\title{
Peritoneal Dissemination: A Pending Issue in Gastric Cancer Worth Exploring
}

\author{
Santiago González-Moreno, MD, PhD \\ Department of Surgical Oncology, Peritoneal Surface Oncology Program, Centro Oncológico M. D. Anderson \\ International España, Madrid, Spain
}

Gastric cancer is a peritoneal-borne disease. Although our attention usually first turns to regional lymphatic extension in cases of localized disease, we know that most gastric adenocarcinomas will eventually spread to the peritoneum sooner or later in their natural history, even in the absence of lymph node involvement. Moreover, peritoneal carcinomatosis is a common manifestation of recurrent disease after curative-intent resection, even when the most efficacious adjuvant or neoadjuvant treatments have been used. The adverse prognostic effect of peritoneal dissemination in this disease is well acknowledged, no matter whether it consists of established macroscopic implants or isolated free cancer cells (detected by peritoneal washing cytology) with no overt peritoneal carcinomatosis. In both cases, current treatment guidelines call for palliative therapeutic measures based on systemic chemotherapy.

Much progress has occurred in recent years in the adjuvant and neoadjuvant treatment of locally advanced gastric cancer, leading to changes in practice across the United States and Europe. Promising reports on the management of peritoneal carcinomatosis from gastric cancer have also been produced, both by Yonemura et al. in Japan and Glehen et al. in France, although these have not attracted as much attention. ${ }^{1,2}$ The authors of these studies concur that a complete resection of all visible disease (primary tumor and peritoneal implants), combined with hyperthermic intraperitoneal chemotherapy (HIPEC), offer a chance for long-term survival in this adverse scenario. Although these are neither randomized nor large-size trials, they call our attention to the fact that progress in the

(C) Society of Surgical Oncology 2009

Published Online: 24 September 2009

S. González-Moreno, MD, $\mathrm{PhD}$

e-mail: sgonzalez@mdanderson.es management of peritoneal carcinomatosis from gastric cancer is likewise possible and worth exploring.

Okabe and colleagues have pursued this task as well. Their study in the present issue of Annals of Surgical Oncology adds to the expectations created by the aforementioned phase II efforts. ${ }^{3}$ The strategy evaluated by Okabe et al. consists of radical surgery for the primary tumor after adequate response of the peritoneal disease to two cycles of systemic chemotherapy with $\mathrm{S}-1$ and cisplatin (a common regimen for disseminated gastric cancer in Japan), as assessed at surgical exploration. Those patients with a complete peritoneal response (overall 46\%) showed a survival advantage over those who did not respond. Resection of residual peritoneal disease (if present) or of posttreatment peritoneal scarring was not pursued. They report an impressive 43-month median survival in the group of patients who underwent an $\mathrm{R} 0$ resection, as well as a small but real number of patients who remained free of disease after 4 years. Although this is a small retrospective study of 41 patients with various degrees of peritoneal involvement, the bottom line is that a subset of patients with peritoneal spread-macroscopic, microscopic, or bothwho are candidates only for palliative chemotherapy by present standards, may become long-term survivors when treated by a curative-intent approach that combines induction systemic chemotherapy and radical surgery. The data of the Okabe study add to that of the Yonemura and Glehen studies in underlining two important facts: a complete surgical clearance of all macroscopic disease is an absolute requirement for improved survival, and the more limited the peritoneal disease volume and extension, the more likely it is that this goal may be achieved.

The strategy of using induction chemotherapy in preparation for radical resection in gastric cancer with peritoneal seeding has already been reported by Yonemura et al. and is a recognized line of clinical research in this 
field. ${ }^{4}$ Yonemura and colleagues use iterative cycles of neoadjuvant concurrent intraperitoneal and systemic chemotherapy (the so-called NIPS approach) with the aim of chemically cytoreducing the macroscopic peritoneal disease and eliminating the microscopic free cancer cell component, thus maximizing the chances of achieving a complete cytoreduction. A negative peritoneal cytology is an absolute requirement to submit these patients for cytoreductive surgery and HIPEC, according to the work of Yonemura and colleagues. The unfavorable prognostic effect of a positive peritoneal cytology, regardless of the presence or absence of peritoneal implants ( $p$ status), is again stressed in the study of Okabe and colleagues by the dramatic difference in response to the induction chemotherapy between cytology-negative (82\%) and cytologypositive cases $(37 \%)$, and ultimately in survival. The message seems clear that free cancer cells in the peritoneal cavity, if present, need to be specifically targeted in order to make a difference in the outcome of gastric cancer patients with peritoneal spread.

From a surgical oncologist perspective, dealing with patients with peritoneal seeding from gastric cancer is difficult, demanding, and often unrewarding. However, these patients are not scarce in our practices. Peritoneal dissemination from gastric cancer can be treated, and better outcomes than those provided by systemic chemotherapy alone are possible in selected patients. Our enemies are invisible free cancer cells as much as widespread macroscopic implants. Our tools include radical surgery and a chemotherapeutic targeting of the peritoneal surfaces without neglecting systemic disease control. Studies like that of Okabe and colleagues, even if retrospective and small in size, have their value as they join others in the exploration of new strategies that can provide the glimmer of hope sought by Sugarbaker and Yonemura in the management of this difficult disease. ${ }^{5}$ They provide the bases upon which larger prospective trials can be designed. There are many questions to be answered, the most important being how to identify that subset of patients who will benefit most from a radical treatment approach. Following this line, a multicentric prospective phase II trial exploring the combination of NIPS followed by cytoreductive surgery and HIPEC plus subsequent systemic chemotherapy is being conducted by the Spanish Peritoneal Surgical Oncology Group (GECOP), with the aim of making further progress in the management of peritoneal seeding from gastric cancer.

\section{REFERENCES}

1. Yonemura Y, Kawamura T, Bandou E, et al. Treatment of peritoneal dissemination from gastric cancer by peritonectomy and chemohyperthermic peritoneal perfusion. Br J Surg. 2005;92: $370-5$.

2. Glehen O, Schreiber V, Cotte R, et al. Cytoreductive surgery and intraperitoneal chemohyperthermia for peritoneal carcinomatosis arising from gastric cancer. Arch Surg. 2004;139:20-6.

3. Okabe H, Ueda S, Obama K, et al. Induction chemotherapy with S-1 plus cisplatin followed by surgery for treatment of gastric cancer with peritoneal dissemination. Ann Surg Oncol. DOI: 10.1245/s10434-009-0706-z.

4. Yonemura Y, Bandou E, Sawa T, et al. Neoadjuvant treatment of gastric cancer with peritoneal dissemination. Eur J Surg Oncol. 2006;32:661-5.

5. Sugarbaker PH, Yonemura Y. Palliation with a glimmer of hope: management of resectable gastric cancer with peritoneal carcinomatosis. Hepatogastroenterology. 2001;48:1238-47. 